\title{
SELEÇÃO DE MARCADORES PARA A BUSCA ATIVA DE ENDOFTALMITE APÓS CIRURGIA DE CATARATA
}

\author{
Selection of markers for active search of endophthalmitis after cataract surgery
}

Selección de marcadores para la búsqueda activa de endoftalmitis tras cirugía de catarata

Reginaldo Adalberto Luz ${ }^{1 *}$, Tadeu Cvintal'2, Edney Cabral Silva², Maria Clara Padoveze ${ }^{3}$

RESUMO: Objetivo: Identificar marcadores potenciais para auxiliar na busca ativa de endoftalmite após cirurgia de catarata. Método: Estudo retrospectivo, descritivo e longitudinal, realizado por meio da revisão de prontuários de pacientes submetidos à cirurgia de catarata. Resultados: A amostra incluiu 20 pacientes (grupo de estudo - GE) que desenvolveram endoftalmite pós-operatória e 309 pacientes (grupo controle — GC) que não apresentaram endoftalmite. Os dados foram analisados para identificar os marcadores clínicos e epidemiológicos com uma diferença percentual $\geq 30 \%$ entre os grupos. Em comparação com o GC, o GE teve frequência $\geq 30 \%$ em: sinais e sintomas pós-operatórios definidos (dor, reação da câmara anterior, hipópio, edema da córnea, hiperemia conjuntival e opacidade vítrea); mais de 4 retornos pós-operatórios; e realização de injeção de antibiótico intravítreo. Conclusão: Os indicadores selecionados são sugeridos para incorporação na busca ativa das infecções pós-operatórias de endoftalmite, visando à facilidade operacional do sistema de vigilância epidemiológica. Palavras-chave: Controle de infecções. Enfermagem. Extração de catarata. Endoftalmite. Infecção da ferida cirúrgica.

ABSTRACT: Objective: To identify potential markers to assist in the active search of endophthalmitis after cataract surgery. Method: Retrospective, descriptive, and longitudinal study, conducted through review of medical records of patients who underwent cataract surgery. Results: The sample included 20 patients (study group - SG) who developed postoperative endophthalmitis and 309 patients (control group - CG) who did not have endophthalmitis. The data were analyzed to identify the clinical and epidemiological markers with a percentage difference $\geq 30 \%$ between groups. In comparison with the CG, the SG demonstrated frequency $\geq 30 \%$ in established postoperative signs and symptoms (pain, anterior chamber reaction, hypopyon, corneal edema, conjunctival hyperemia, and vitreous opacity); more than four postoperative appointments; and administration of an intravitreal antibiotic injection. Conclusion: The selected markers are suggested for incorporation into the active search for post-operative endophthalmitis, in order to facilitate the operation of the epidemiological surveillance system.

Keywords: Infection control. Nursing. Cataract Extraction. Endophthalmitis. Surgical wound infection.

RESUMEN: Objetivo: Identificar marcadores potenciales para auxiliar en la búsqueda activa de endoftalmitis tras cirugía de facectomía. Método: Estudio retrospectivo, descriptivo y longitudinal, realizado por medio de la revisión de prontuarios de pacientes sometidos a la cirugía de catarata. Resultados: La muestra incluyó 20 pacientes (grupo de estudio - GE) que desarrollaron endoftalmitis pos-operatoria y 309 pacientes (grupo control — GC) que no presentaron endoftalmitis. Los datos fueron analizados para identificar los marcadores clínicos y epidemiológicos con una diferencia porcentual $\geq 30 \%$ entre los grupos. En comparación con el GC, el GE tuvo frecuencia $\geq 30 \%$ en: señales y síntomas pos-operatorios definidos (dolor, reacción de la cámara anterior, hipopión, edema de la córnea, hiperemia conjuntival y opacidad vítrea); más de 4 retornos pos-operatorios; y realización de inyección de antibiótico intravítreo. Conclusión: Los indicadores seleccionados son sugeridos para incorporación en la búsqueda activa de las infecciones pos-operatorias de endoftalmitis, buscando la facilidad operacional del sistema de vigilancia epidemiológica.

Palabras clave: Control de infecciones. Enfermería. Extracción de catarata. Endoftalmitis. Infección de la herida quirúrgica.

'Mestre pela Escola de Enfermagem da Universidade de São Paulo (USP); professor na Faculdade de Ciências Médicas da Santa Casa de São Paulo - São Paulo (SP), Brasil. ${ }^{2}$ Médico oftalmologista do Instituto de Oftalmologia Tadeu Cvintal - São Paulo (SP), Brasil.

${ }^{3}$ Professora doutora do Departamento de Enfermagem em Saúde Coletiva da Escola de Enfermagem da USP - São Paulo (SP), Brasil.

*Autor correspondente: reginaldo.enfermeiro@gmail.com

Recebido: 05/01/2018 - Aprovado: 21/04/2018

DOI: $10.5327 / Z 1414-4425201800030005$ 


\section{INTRODUÇÃO}

A cirurgia de correção de catarata, ou facectomia, está associada a várias complicações pós-operatórias, incluindo a infecção intraocular, conhecida como endoftalmite. Os sinais clínicos de endoftalmite são frequentemente observados nos primeiros sete dias após a cirurgia ${ }^{1,2}$. Os sinais e sintomas mais comuns são: reação da câmara anterior, hipópio, edema de pálpebras e da córnea $a^{3-5}$, hiperemia conjuntival e vítreo turvo ${ }^{1,4,6}$. Em muitos casos, os pacientes se queixam de dor ocular ou baixa acuidade visual ${ }^{3-5,7}$.

A incidência média de endoftalmite pós-operatória (EPO) é de $0,13 \%^{1,8-11}$. No entanto, a ocorrência em cenário de surto pode ser devastadora, devido ao grande número de cirurgias de catarata normalmente realizadas em um único dia, expondo os pacientes a fatores de risco similares ${ }^{12-14}$. A maioria dos pacientes que desenvolve endoftalmite evolui para deficiência visual ou cegueira ${ }^{1,5,7,13,14}$, não sendo rara a necessidade da retirada do globo ocular ou de seu conteúdo interno, através dos procedimentos de enucleação ${ }^{14}$ ou evisceração ${ }^{13,15}$, respectivamente.

Em muitos estabelecimentos de saúde onde a facectomia é realizada, o profissional responsável pelo sistema de vigilância da endoftalmite é um enfermeiro. A vigilância da EPO é, muitas vezes, desafiadora, por duas razões principais. Em primeiro lugar, muitos cirurgiões não relatam os casos de infecção; em segundo, as características da apresentação clínica da endoftalmite podem ser parecidas com a síndrome tóxica do segmento anterior ${ }^{16,17}$ e alguns casos podem não ser diagnosticados corretamente, levando a taxas imprecisas.

Para evitar a subnotificação de endoftalmites, é necessário o desenvolvimento de uma estrutura e de processos de trabalho para uma vigilância epidemiológica, além da seleção de marcadores que apontem os casos suspeitos e que aumentem a sensibilidade e a especificidade na detecção de casos.

\section{OBJETIVO}

Identificar as características clínicas e epidemiológicas dos casos de endoftalmite após cirurgias de catarata, a fim de indicar os marcadores mais apropriados para apoiar a busca ativa dos casos de endoftalmites.

\section{MÉTODO}

Trata-se de um estudo retrospectivo, descritivo e longitudinal, realizado por meio da revisão de prontuários de pacientes submetidos à cirurgia de correção de catarata/facectomia. O estudo foi realizado em uma instituição filantrópica que atende exclusivamente pacientes do Sistema Único de Saúde (SUS), localizada no município de São Paulo, Brasil.

Os prontuários de 329 pacientes foram classificados em dois grupos: grupo controle (GC; $\mathrm{n}=309$ ), que incluiu os pacientes submetidos à cirurgia de catarata de maio a junho de 2013 sem EPO; e grupo de estudo (GE; $n=20$ ), que incluiu pacientes submetidos à cirurgia de catarata de abril de $2010 \mathrm{a}$ fevereiro de 2013 com EPO. O GE exigiu um maior período de coleta de dados, em comparação com o GC, devido à baixa incidência de EPO.

Para calcular o tamanho da amostra, o diagnóstico de EPO foi escolhido como desfecho primário. O número de participantes no GC necessitou ser 15 vezes maior que o do GE para detectar variáveis clínicas com uma diferença percentual igual ou superior a $30 \%$ entre os grupos. Esse valor da diferença percentual foi arbitrado pelos pesquisadores para selecionar apenas aquelas variáveis com maior relevância. Com a definição de um nível de significância de $5 \%$ e o poder do teste de $85 \%$, o tamanho da amostra necessário foi de 20 pacientes no GE e 309 pacientes no GC, para detectar essa diferença estabelecida.

\section{Coleta de dados}

As características demográficas dos pacientes foram coletadas de todos os prontuários selecionados, usando um formulário padronizado. Os dados clínicos para comparações entre os grupos foram coletados desde o primeiro dia após a cirurgia até aproximadamente dois meses, período em que são realizados os três retornos programados para o acompanhamento pós-operatório de cirurgias de catarata.

\section{Definição do caso}

A EPO foi definida como uma infecção intraocular diagnosticada em até seis semanas após a cirurgia, caracterizada pela ocorrência de pelo menos três dos seguintes sinais ou sintomas: diminuição da acuidade visual, dor ocular, hipópio, reação da câmara anterior, vítreo turvo ou hiperemia de conjuntiva. 


\section{Rotina de acompanhamento pós-operatório}

Todos os pacientes foram avaliados pela equipe cirúrgica no primeiro dia pós-operatório, nove dias após o primeiro retorno e um mês após o segundo retorno. O diagnóstico clínico de endoftalmite foi confirmado por um especialista em retina, após o diagnóstico diferencial da síndrome do segmento anterior tóxico. A medida da acuidade visual foi realizada usando o teste de Snellen, onde $20 / 20$ representa $100 \%$ de visão central, 20 / 40 representa $85 \%, 20 / 200$ representa $20 \%$ e $20 / 400$ representa $10 \%$. Para a visão central pior do que $10 \%$, as seguintes categorias foram aplicadas, da visão mais alta para a mais baixa: conta dedos (ou seja, a capacidade de contar dedos a uma determinada distância, relatada em metros), movimento de mãos, percepção da luz e sem percepção da luz ${ }^{18}$. Os dados dos casos suspeitos de endoftalmite foram registrados pelo enfermeiro de controle de infecção, usando um formulário padronizado.

\section{Considerações éticas}

O estudo foi aprovado pelo Comitê de Ética em Pesquisa da Escola de Enfermagem da Universidade de São Paulo, Brasil (número de aprovação CAAE 01039912.3.0000.5392). O anonimato dos pacientes foi mantido.

\section{Análise estatística}

Uma análise descritiva foi realizada, utilizando o software Epi Info, versão 7.1.2.0 (Centers for Disease Control and Prevention, Atlanta, Geórgia, Estados Unidos). Os dados foram expressos como médias, valores mínimo e máximo e desvio padrão (DP). O teste $t$ de Student foi usado para comparar as médias, quando aplicável.

\section{RESULTADOS}

\section{Grupo controle: caracterização}

O GC ( $\mathrm{n}=309)$ foi composto por $192(62,1 \%)$ mulheres e $117(37,9 \%)$ homens. A idade média foi de 68,3 anos, (intervalo, 41-95; DP 9,6); 140 (45,3\%) tinham mais de 70 anos. O número médio de retornos pós-operatórios dos pacientes sem endoftalmite foi de 3,2 (intervalo, 3-7; DP 0,6), em que 297 (96,1\%) deles necessitaram, no máximo, de 4 visitas de acompanhamento dentro de 2 meses após a cirurgia. Três pacientes necessitaram de revisão cirúrgica, devido a complicações pós-operatórias que não endoftalmite, incluindo reposicionamento da lente intraocular, sutura corneana ou vitrectomia anterior.

\section{Grupo controle: evolução pós-operatória}

No primeiro dia após a cirurgia, mais de metade dos pacientes apresentaram reação da câmara anterior, edema da córnea, hiperemia de conjuntiva e dobras de membrana de Descemet; córnea nebulosa foi observada em 138 (44,7\%) pacientes e edema de pálpebras, em 98 (31,7\%). Outra complicação menos comum foi a injeção ciliar ( $0,3 \%)$; hipópio esteve presente em menos de $2 \%$ dos pacientes; e vítreo turvo não foi observado. A dor ocular foi relatada por $82(26,5 \%)$ pacientes (Tabela 1).

O segundo retorno para acompanhamento ocorreu, em média, 9 dias após a cirurgia (intervalo, 5-17; DP 2), com a apresentação de menos sinais e sintomas, quando comparados

Tabela 1. Sinais observados e sintomas relatados pelos pacientes no primeiro dia após a cirurgia de catarata. São Paulo, 2017 ( $n=329$ ).

\begin{tabular}{|l|c|c|c|c|c|c|}
\hline \multirow{2}{*}{ Sinais e sintomas } & \multicolumn{2}{|c|}{ Sim } & \multicolumn{2}{|c|}{ Não } & \multicolumn{2}{c|}{ NI } \\
\cline { 2 - 7 } & $\mathbf{n}$ & $\%$ & $\mathbf{n}$ & $\%$ & $\mathbf{n}$ & $\%$ \\
\hline RCA & 251 & 81 & 48 & 16 & 10 & 3,2 \\
\hline Injeção ciliar & 47 & 15 & 228 & 74 & 34 & 11 \\
\hline Córnea nebulosa & 138 & 45 & 156 & 51 & 15 & 4,9 \\
\hline Hiperemia conjuntival & 184 & 60 & 119 & 39 & 06 & 1,9 \\
\hline Desepitelização da córnea & 22 & 7,1 & 270 & 87 & 17 & 5,5 \\
\hline Edema de córnea & 209 & 68 & 98 & 32 & 02 & 0,6 \\
\hline DMD & 159 & 52 & 148 & 48 & 02 & 0,6 \\
\hline Dor ocular & 82 & 27 & 221 & 72 & 06 & 1,9 \\
\hline Edema de pálpebra & 98 & 32 & 200 & 65 & 11 & 3,6 \\
\hline Hifema & 04 & 1,3 & 302 & 98 & 03 & 1 \\
\hline Hipópio & 05 & 1,6 & 303 & 98 & 01 & 0,3 \\
\hline Ml na LIO & 06 & 1,9 & 297 & 96 & 06 & 1,9 \\
\hline Precipitados ceráticos & 05 & 1,6 & 298 & 96 & 06 & 1,9 \\
\hline Seidel & 15 & 4,9 & 281 & 91 & 13 & 4,2 \\
\hline Vítreo turvo & - & - & 303 & 98 & 06 & 1,9 \\
\hline Trave vítrea & 01 & 0,3 & 303 & 98 & 05 & 1,6 \\
\hline Vitreíte & - & - & 304 & 98 & 05 & 1,6 \\
\hline
\end{tabular}

NI: não informado; RCA: reação de câmara anterior; DMD: dobras na membrana de Descemet; MI: membrana inflamatória; LIO: lente intraocular. 
ao retorno para acompanhamento no primeiro dia pós-operatório. As complicações mais comuns foram: dobras de membrana de Descemet ( $\mathrm{n}=108 ; 35 \%)$ e córnea nebulosa $(\mathrm{n}=105 ; 34 \%)$, seguidas de edema corneano $(\mathrm{n}=62 ; 20,1 \%)$ e reação da câmara anterior ( $\mathrm{n}=57 ; 18,4 \%)$. Outras complicações estavam presentes em menos de $2,5 \%$ dos pacientes (Tabela 2). No final do período de seguimento pós-operatório, $221(71,5 \%)$ pacientes apresentaram uma acuidade visual final (AVF) de 20/50 ou melhor.

\section{Grupo de estudo: caracterização}

O GE $(\mathrm{n}=20)$ incluiu $12(60 \%)$ mulheres e $8(40 \%)$ homens. A idade média foi de 67,5 anos (intervalo, 47-83; DP 8,8); 13 (65\%) deles tinham mais de 65 anos. O número médio de retornos para acompanhamento pós-operatório dos pacientes com endoftalmite foi de 14,5 (intervalo 8-25; DP 4,6). Entre as intervenções necessárias para o tratamento da endoftalmites, todos os pacientes receberam injeção de antibiótico intravítrea, realizadas em um centro cirúrgico. Treze (65\%) pacientes necessitaram de 2 revisões cirúrgicas e 3 pacientes necessitaram de mais de 2 revisões cirúrgicas.

\section{Grupo de estudo: evolução pós-operatória}

Os sinais e sintomas mais comumente observados (>50\%) no momento do diagnóstico foram: reação da câmara anterior, córnea nebulosa, edema corneano, hiperemia de conjuntiva e hipópio. Vítreo turvo e dobras da membrana de Descemet estavam presentes em 50 e $40 \%$ dos casos, respectivamente. Menos de $30 \%$ dos pacientes apresentaram precipitados ceráticos, membrana inflamatória sobre a lente intraocular e edema das pálpebras, dentre outros. A dor ocular foi relatada por 14 (70\%) pacientes (Tabela 2$)$.

O número médio de dias entre a data da cirurgia e o diagnóstico de endoftalmite foi de 7,5 (intervalo 1-30; DP 8,7$)$. A maioria dos pacientes $(n=11 ; 55 \%)$ foi diagnosticada após o terceiro dia pós-operatório. A acuidade visual pós-operatória foi melhor do que 20 / 60 para apenas $4(20 \%)$ pacientes e, em 8 (40\%) casos a acuidade visual

Tabela 2. Sinais observados e sintomas relatados pelos pacientes após cirurgia de catarata de acordo com a presença ou ausência de endoftalmite. São Paulo, 2017 ( $n=329)$.

\begin{tabular}{|c|c|c|c|c|c|c|c|c|c|c|c|c|c|}
\hline \multirow{3}{*}{ Sinais e sintomas } & \multicolumn{6}{|c|}{$\begin{array}{l}\text { Pacientes com endoftalmite* } \\
n=20\end{array}$} & \multicolumn{6}{|c|}{$\begin{array}{l}\text { Pacientes sem endoftalmite } \\
\text { n=309 }\end{array}$} & \multirow{3}{*}{$\begin{array}{l}\text { Diferença } \\
(\%)\end{array}$} \\
\hline & \multicolumn{2}{|c|}{ Sim } & \multicolumn{2}{|c|}{ Não } & \multicolumn{2}{|c|}{ NI } & \multicolumn{2}{|c|}{ Sim } & \multicolumn{2}{|c|}{ Não } & \multicolumn{2}{|c|}{ NI } & \\
\hline & $n$ & $\%$ & $n$ & $\%$ & $n$ & $\%$ & $\mathbf{n}$ & $\%$ & $\mathbf{n}$ & $\%$ & n & $\%$ & \\
\hline RCA & 17 & 85 & 01 & 5 & 02 & 10 & 57 & 18 & 236 & 76 & 16 & 5,2 & 66,6 \\
\hline Injeção ciliar & 03 & 15 & 12 & 60 & 05 & 25 & 01 & 0,3 & 33 & 11 & 275 & 89 & 14,7 \\
\hline Córnea nebulosa & 17 & 85 & 03 & 15 & - & - & 105 & 34 & 191 & 62 & 13 & 4,2 & 51 \\
\hline Hiperemia conjuntival & 12 & 60 & 06 & 30 & 02 & 10 & 07 & 2,3 & 29 & 9,4 & 273 & 88 & 57,7 \\
\hline Desepitelização da córnea & 01 & 5 & 15 & 75 & 04 & 20 & 02 & 0,6 & 57 & 18 & 250 & 81 & 4,4 \\
\hline Edema de córnea & 14 & 70 & 05 & 25 & 01 & 5 & 62 & 20 & 217 & 70 & 30 & 9,7 & 49,9 \\
\hline DMD & 8 & 40 & 10 & 50 & 02 & 10 & 108 & 35 & 145 & 47 & 56 & 18 & 05 \\
\hline Dor ocular & 14 & 70 & 06 & 30 & - & - & 01 & 0,3 & 273 & 88 & 35 & 11 & 69,7 \\
\hline Edema de pálpebra & 04 & 20 & 12 & 60 & 04 & 20 & 01 & 0,3 & 31 & 10 & 277 & 90 & 19,7 \\
\hline Hifema & 01 & 5 & 16 & 80 & 03 & 15 & - & - & 83 & 27 & 226 & 73 & 05 \\
\hline Hipópio & 12 & 60 & 08 & 40 & - & - & - & - & 217 & 70 & 92 & 30 & 60 \\
\hline MI na LIO & 05 & 25 & 12 & 60 & 03 & 15 & 04 & 1,3 & 92 & 30 & 213 & 69 & 23,7 \\
\hline Precipitados ceráticos & 05 & 25 & 11 & 55 & 04 & 20 & 01 & 0,3 & 90 & 29 & 218 & 71 & 24,7 \\
\hline Seidel & 01 & 5 & 19 & 95 & - & - & - & - & 263 & 85 & 46 & 15 & 05 \\
\hline Vítreo turvo & 10 & 50 & 10 & 50 & - & - & 01 & 0,3 & 274 & 89 & 34 & 11 & 49,7 \\
\hline Trave vítrea & 04 & 20 & 16 & 80 & - & - & 03 & 1 & 131 & 42 & 175 & 57 & 19 \\
\hline Vitreíte & 01 & 5 & 18 & 90 & 01 & 5 & 01 & 0,3 & 134 & 43 & 174 & 56 & 4,7 \\
\hline
\end{tabular}

NI: não informado; RCA: reação de câmara anterior; DMD: dobras na membrana de Descemet; MI: membrana inflamatória; LIO: lente intraocular; *relacionado ao dia do diagnóstico (média=7,5 dias; IC 1-30; DP=8,7); ₹relacionado ao segundo retorno para acompanhamento (média=9,1 dias; IC 5-17; DP=2); ${ }^{5}$ \% encontrada nos casos de endoftalmites) - (\% encontrada nos controles). 
variou entre $20 / 60$ e $20 / 200 ; 8$ (40\%) pacientes tinham acuidade visual igual ou pior do que a capacidade de contar os dedos do avaliador (CD). A AVF avaliada após dois meses após a cirurgia foi pior do que $20 / 50$ em 17 (85\%) pacientes. Sete $(35 \%)$ de todos os casos tiveram AVF igual ou pior que CD. Foram coletadas amostras vítreas de 14 $(66,7 \%)$ pacientes. Resultados de cultura positivos foram encontrados em 6 das 14 amostras vítreas, como se segue: Staphylococcus coagulase-negativo $(\mathrm{n}=3)$, Streptococcus spp. $(\mathrm{n}=2)$ e Staphylococcus aureus $(\mathrm{n}=1)$.

\section{Diferenças entre os grupos}

Os sinais e sintomas observados no GE com uma diferença percentual igual ou superior a $30 \%$ no segundo retorno para acompanhamento pós-operatório, em comparação com o GC, foram: dor ocular, reação da câmara anterior, hipópio, hiperemia conjuntival, edema corneano e vítreo turvo (Tabela 2).

Outras diferenças encontradas foram: maior número de retornos para acompanhamento pós-operatório no GE $(\mathrm{p}<0,001)$ e a necessidade de injeções de antibiótico intravítreo, que só foram realizadas no GE.

\section{DISCUSSÃO}

Embora a apresentação clínica da EPO seja bem conhecida, o diagnóstico diferencial da síndrome tóxica do segmento anterior pode ser difícil ${ }^{16}$ e levar à subnotificação dos casos. Assim, o desenvolvimento de ferramentas para a busca ativa dos casos com alta sensibilidade e especificidade para endoftalmite é necessário para uma vigilância epidemiológica apropriada.

Os sinais observados de endoftalmite no GE são consistentes com os achados anteriores de outros autores, que também relataram a presença de hipópio, reação da câmara anterior e edema da córnea ${ }^{3-5}$ como as características clínicas mais frequentes, seguidas de hiperemia conjuntival, vítreo turvo, ${ }^{1,4}$ dor ocular e baixa acuidade visual ${ }^{3-5,7}$. Uma reação inflamatória leve e transitória da câmara anterior observada no primeiro dia após a cirurgia de catarata é comum, de acordo com a literatura ${ }^{3,4}$.

Outros sinais, como edema da córnea, hiperemia conjuntival, dobras na membrana Descemet e nebulosidade da córnea estavam presentes em mais de $40 \%$ dos pacientes no primeiro dia pós-operatório, dificultando o diagnóstico de endoftalmite em sua forma leve e de apresentação precoce. Por esse motivo, foi feita a opção pela comparação das diferenças na apresentação clínica, baseando-se nos resultados do segundo retorno dos pacientes do GC, evitando o viés de incluir potenciais casos falso-positivos. O tempo de início dos sintomas mostrou grande variabilidade. No entanto, os diagnósticos foram realizados, em média, em até uma semana após a cirurgia ${ }^{1,9}$. Vale ressaltar que nem todos os casos clinicamente positivos de endoftalmite foram confirmados por cultura microbiana. Vários estudos apontaram que mais de $40 \%$ das amostras vítreas não apresentaram crescimento microbiano ${ }^{8,9}$. Os microrganismos Gram-positivos foram os agentes etiológicos mais comuns entre os casos em que a identificação do agente foi possível, o que está de acordo com a literatura ${ }^{1,8,9}$.

A AVF apresentou diferença importante entre os grupos. A maioria dos pacientes com endoftalmite apresentou AVF ruim, caracterizada como deficiência visual ou cegueira, consistente com os achados de outros estudos ${ }^{1,5,13,14}$. Após a facectomia, os pacientes do GC necessitaram de poucas revisões cirúrgicas, que não estavam relacionadas a complicações infecciosas. Em contraste, os pacientes com endoftalmite foram submetidos a pelo menos uma e até três revisões cirúrgicas, como consequência direta da evolução do caso, o que está de acordo com estudos que descrevem o tratamento da endoftalmite ${ }^{3,6,711}$. O número de retornos para o acompanhamento pós-operatório de pacientes com endoftalmite (GE) foi significativamente maior do que o GC. No entanto, não identificamos nenhum estudo na literatura comparando o número de visitas de acompanhamento exigidas por pacientes com e sem EPO.

Baseado nos resultados deste estudo, os marcadores mais adequados para a busca ativa de endoftalmite após a cirurgia de catarata incluem: presença de três ou mais dos seguintes sinais e sintomas pós-operatórios: dor ocular, reação da câmara anterior, hipópio, edema corneano, hiperemia conjuntival e vítreo turvo; mais de quatro retornos para o acompanhamento dentro de dois meses após a cirurgia; e indicação médica para injeção de antibiótico intravítreo.

\section{CONCLUSÕES}

Os indicadores selecionados - presença de três ou mais sinais e sintomas pós-operatórios (dor ocular, reação da câmara anterior, hipópio, edema corneano, hiperemia conjuntival e vítreo turvo); mais de quatro retornos para o acompanhamento 
dentro de dois meses após a cirurgia; e indicação médica para injeção de antibiótico intravítreo — são sugeridos para incorporação na busca ativa de endoftalmite pós-operatória, visando à facilidade operacional do sistema de vigilância epidemiológica, potencialmente ampliando a sensibilidade e a especificidade do sistema.
O conhecimento dos sinais e sintomas relevantes da endoftalmite (dor ocular, reação da câmara anterior, hipópio, edema corneano, hiperemia conjuntival e vítreo turvo) também favorece o desempenho dos enfermeiros no seu papel de orientação e apoio aos pacientes e na contribuição para o diagnóstico e o tratamento precoce dessa infecção.

\section{REFERÊNCIAS}

1. Raen M, Sandvik GF, Drolsum L. Endophthalmitis following cataract surgery: the role of prophylactic postoperative chloramphenicol eye drops. Acta Ophthalmol. 2013;91(2):118-22. https://doi. org/10.1111/j.1755-3768.2011.02324.x

2. Mattos FB, Saraiva FP, Angotti-Neto H, Passos AF. Outbreak of ochrobactrum anthropi endophthalmitis following cataract surgery. J Hosp Infect. 2013;83(4):337-40. https://doi.org/10.1016/j. jhin.2012.11.027

3. Lalitha P, Das M, Purva PS, Karpagam R, Geetha M, Lakshmi Priya J, et al. Postoperative endophthalmitis due to Burkholderia cepacia complex from contaminated anaesthetic eye drops. $\mathrm{Br}$ J Ophthalmol. 2014;98(11):1498-502. https://doi.org/10.1136/ bjophthalmol-2013-304129

4. Ji Y, Jiang C, Ji J, Luo Y, Jiang Y, Lu Y. Post-cataract endophthalmitis caused by multidrug-resistant Stenotrophomonas maltophilia: clinical features and risk factors. BMC Ophthalmol. 2015;15:14. https://doi. org/10.1186/1471-2415-15-14

5. Rishi E, Rishi P, Sengupta S, Jambulingam M, Madhavan HN, Gopal L, et al. Acute postoperative Bacillus cereus endophthalmitis mimicking toxic anterior segment syndrome. Ophthalmology. 2013;120(1):1815. https://doi.org/10.1016/j.ophtha.2012.07.009

6. Kelkar AS, Kelkar JA, Barve PM, Mulay A, Sharma S, Amoaku W. Post-clear corneal phacoemulsification endophthalmitis: profile and management outcomes at a tertiary eye care center in western India. J Ophthalmic Inflamm Infect. 2016;6(1):48. https://doi.org/10.1186/ s12348-016-0115-y

7. Vinekar A, Dogra MR, Avadhani K, Gupta V, Gupta A, Chakrabarti A. Management of recurrent postoperative fungal endophthalmitis. Indian J Ophthalmol. 2014;62(2):136-40. https:// doi.org/10.4103/0301-4738.128588

8. Rahman N, Murphy CC. Impact of intracameral cefuroxime on the incidence of postoperative endophthalmitis following cataract surgery in Ireland. Ir J Med Sci. 2015;184(2):395-8. https://doi.org/10.1007/ s11845-014-1127-y

9. Shorstein NH, Winthrop KL, Herrinton LJ. Decreased postoperative endophthalmitis rate after institution of intracameral antibiotics in a Northern California eye department. J Cataract Refract Surg. 2013;39(1):8-14. https://doi.org/10.1016/j.jcrs.2012.07.031
10. Kessner R, Golan S, Barak A. Changes in the etiology of endophthalmitis from 2003 to 2010 in a large tertiary medical center. Eur J Ophthalmol. 2014;24(6):918-24. https://doi.org/10.5301/ejo.5000473

11. Creuzot-Garcher C, Benzenine E, Mariet AS, de Lazzer A, Chiquet C, Bron AM, et al. Incidence of acute postoperative endophthalmitis after cataract surgery: a nationwide study in France from 2005 to 2014. Ophthalmology. 2016;123(7):1414-20. https://doi.org/10.1016/j. ophtha.2016.02.019

12. Gautam P, Joshi SN, Thapa M, Sharma A, Shah DN. Outcome of the patients with post-operative cluster endophthalmitis referred to a tertiary level eye care center in Nepal. Nepal J Ophthalmol. 2013;5(2):235-41. https://doi.org/10.3126/nepjoph.v5i2.8735

13. Bhat SS, Undrakonda V, Mukhopadhyay C, Parmar PV. Outbreak of multidrug-resistant acute postoperative endophthalmitis due to Enterobacter aerogenes. Ocul Immunol Inflamm. 2014;22(2):121-6. https://doi.org/10.3109/09273948.2013.830752

14. Buchta V, Feuermannová A, Vása M, Basková L, Kutová R, Kubátová A, et al. Outbreak of fungal endophthalmitis due to Fusarium oxysporum following cataract surgery. Mycopathologia. 2014;177(1-2):115-21. https://doi.org/10.1007/s1 1046-013-9721-5

15. Ng AL, Tang WW, Li PS, Li KK. Intracameral cefuroxime in the prevention of postoperative endophthalmitis: an experience from Hong Kong. Graefes Arch Clin Exp Ophthalmol. 2016;254(10):198792. https://doi.org/10.1007/s00417-016-3473-0

16. Lee MH, Cugley D, Atik A, Ang GS. Endophthalmitis or toxic anterior segment syndrome? Clin Exp Optom. 2017;100(1):94-5. https://doi. org/10.1111/cxo.12426

17. Luz RA, Padoveze MC, Souza RQ, Graziano KU, Cvintal T. Toxic anterior segment syndrome after cataract surgery: the implications for nursing. Rev SOBECC. 2015;20(2):96-103. https://doi.org/10.5327/ Z1414-4425201500020005

18. Holladay JT. Visual acuity measurements. J Cataract Refract Surg. 2004;30(2):287-90. https://doi.org/10.1016/j.jcrs.2004.01.014

19. Jeong SH, Cho HJ, Kim HS, Han JI, Lee DW, Kim CG, et al. Acute endophthalmitis after cataract surgery: 164 consecutive cases treated at a referral center in South Korea. Eye (Lond). 2017;31(10):1456-62. https://doi.org/10.1038/eye.2017.85 